\title{
Experimental Investigation of Classification Algorithms for Predicting Lesion Type on Breast DCE-MR Images
}

\author{
D. Janaki Sathya \\ Research Scholar, \\ EEE Department \\ Karpagam University \\ Coimbatore -21
}

\author{
K. Geetha \\ Professor and HOD \\ IT Department \\ Karpagam Institute of Technology
}

\begin{abstract}
Timely revealing of breast cancer is one of the most important issues in determining prognosis for women with malignant tumors. Dynamic contrast-enhanced (DCE) MRI is being increasingly used in the clinical setting to help detect and characterise tissue, suspicious for malignancy and has been shown to be the most sensitive modality for screening highrisk women. Computer-assisted evaluation (CAE) systems have the potential to assist radiologists in the early detection of cancer. A crucial module of the development of such a CAE system will be the selection of an appropriate classification function responsible for separating malignant and benign lesions. The motivation of this paper is to provide qualitative evaluation of three advanced classifiers like artificial neural network, support vector machine and artificial bee colony optimization algorithm trained neural network are being developed for classification of the suspicious lesions in breast MRI. A comparative study of these techniques for lesion classification is made to identify relative merits. As a result, the paper concluded that the neural network trained by artificial bee colony optimization algorithm based classifier outperforms all other explored classifiers for the examined dataset of breast DCE -MR images.
\end{abstract}

\section{Keywords}

Artificial Intelligence, Dynamic Contrast Enhanced Magnetic Resonance Images (DCE-MRI), Artificial Neural Networks, Support Vector Machine, Artificial Bee Colony Optimization, Statistical Texture Features and Mass Classification.

\section{INTRODUCTION}

The accurate finding and classification of diseases, especially on cancers, which are very important in medical science, are difficult to achieve. Accurate classification allows doctors to select suitable therapies and treatment for diseases. Over the last several decades, cancer classification has been advanced, but still has limitations caused by traditional method for morphological appearance analysis [1]. Magnetic resonance is widely used in recent years as a valuable technique in surgical and clinical environment. MR imaging has become a useful modality since it provides plentiful image information and high sensitivity. MRI characteristics play crucial role in medical clinical diagnosis, providing abundant information of the tissues. Breast magnetic resonance (MR) imaging continues to become an important component of the clinical work-up of patients suspected of having breast cancer. The dynamic MR imaging has emerged as a modality that is possibly complementary to mammography and ultrasonography because of the additional three-dimensional spatial and temporal information about the lesion that it yields. Breast MRI has a high sensitivity for breast cancer detection reported as high as $94-100 \%$, but a lower specificity, reported as $37-97 \%$ [2].
It is important to develop CAE (Computer-Assisted Evaluation) based classification techniques to analyze and discriminate the detected and recognize suspicious lesions on breast DCE-MR images. CAE systems are useful for supporting radiologists in detecting and assessing suspicious tissue regions in DCE-MRI data are expected to improve the reliability of clinical decisions and, therewith, to potentially decrease the number of unnecessary biopsies. Significant research has been conducted on the application of computer classification methods to the analysis of breast MR images. The artificial neural networks (ANN) have been one of the most common approaches for researching the classification of malignant and benign breast MR lesions [1, 3-9, 20-22, 2428]. The self-organizing map based artificial neural networks approach is used to assist in the separation of malignant and benign breast MR lesions [10]. Linear discriminant analysis, a typical classification approach has also been applied to breast MR image analysis $[11,12]$. In recent times it has been shown that SVMs outperform a variety of other machine learning techniques when applied to the separation of malignant and benign DCE-MR breast lesions [13, 14, 30, 32].

Computer-assisted evaluation (CAE) system that can automatically analyze lesion features to differentiate between malignant and benign lesions would be very useful. This paper emphasizes on the analysis and usage of different advanced automated classification techniques such as artificial neural networks, support vector machines and artificial bee colony optimization algorithm trained neural network. The proposed model includes the following phases in its CAE system they are feature extraction, feature selection and mass classification. Which is developed to classify mass into benign and malignant based on the statistical textural features extracted from the ROI of breast DCE-MR imaging and feature selection using the statistical hypothesis t-test described in [15], is used to select the set of effective features for the classifier to obtain high accuracy. In this proposed study our specific goal was to assess the performance of the proposed three classifiers for differentiation of breast lesions on breast DCE-MR images in terms of sensitivity, specificity, accuracy and efficiency. The neural network trained by a bee colony optimization algorithm based classifier provides significantly improved specificity, efficiency and overall accuracy for breast cancer detection compared to the other classifiers discussed.

The paper is organized as follows: Section 2 presents a description on analysis of the mass classification model. Section 3 presents the brief review of the proposed classifiers for mass classification. Section 4 describes results and discussion. The conclusion is summed up in Section 5. 


\section{ANALYSIS OF THE MASS CLASSIFICATION MODEL}

The Mass classification model is used for classification on region of interest (ROI) that contains mass on breast DCEMR imaging. To report the task of accuracy and efficiency in interpretation of breast MRI, a Computer-assisted evaluation (CAE) system that can automatically analyze lesion features to differentiate between malignant and benign lesions would be very useful. The proposed model includes the following phases in its CAE system: feature extraction, feature selection and mass classification. Such CAE tools present to the physician both a qualitative and a quantitative description of the disease.

\subsection{Feature Extraction and Selection}

The most important phase of mass classification CAE system is the feature extraction and selection. Features are used as inputs to classifiers that assign them to the class that they represent. The features can be calculated from the ROI characteristics such as the size, shape, density, texture and smoothness of borders, etc. The implemented feature extraction procedure relies on the exploitation of the textural characteristics of the extracted mass. Textures represent tonal variations in the spatial domain and determine the overall visual smoothness or coarseness of image features. They provide important information about the structural arrangements of the objects in the image and their relationship to the environment.

The extraction methods of texture feature play very important role in detecting abnormalities present in breast DCE-MRI. Texture analysis approaches can be summarized into four texture modeling methods: statistical methods, geometrical methods, signal processing methods and model based methods [16]. The statistical textures are found to be best for image classification [17]. In this proposed work, a set of 18 features were extracted from the ROI, fourteen features of GLCM as defined in [18] and four GLHM features, it is possible to calculate textural properties from them and has been proved to be effective for images classification [19].

The above described features were extracted using a window of size $16 \times 16$ pixels with 16 pixels shift. The features extracted are: Energy measure, correlation, inertia, entropy, difference moment, inverse difference moment, sum entropy, difference entropy, sum variance, sum average, difference variance, difference average, information measure of correlation, standard deviation, mean, variance, skewness and kurtosis.

Using excessive features may degrade the performance of the classification algorithm and increase the complexity of the classifier. Some redundant features should be removed to improve the performance of the classifier. Feature selection has been widely used to improve prediction accuracy of classifiers. The improvement in prediction is related to the redundant features or noisy features in the data which can be eliminated by feature selection, relatively few features used in a classifier can keep the classification performance robust.

The hypothesis test is applied to decide whether the feature can discriminate or not. The statistical hypothesis t-test is used. It performs a test of the hypothesis that the data in the vector of data set comes from a distribution with mean zero and returns the result of the test. If the result equal zero it indicates that the null hypothesis (mean is zero) cannot be rejected at the $20 \%$ significance level. If the result equal one it indicates that the null hypothesis can be rejected at the $20 \%$ level. The test indicates that only seven features can discriminate between the two clusters they are: Energy, entropy, mean, variance, skewness, standard deviation and kurtosis. The feature extraction and selection are a key step in mass detection since the performance of CAE depends on both the optimization of the feature selection and the classification method.

\subsection{Mass Type Classification}

Once a proper representation in a feature space has been chosen for the patterns, a classifier should be trained and tested on the data. The role of the classifier is to implement a decision rule that will indicate to which class a given pattern belongs. In other words, it operates a discriminant function on the data. In the two-class case, the classifier creates a decision boundary in the feature space on which the discriminant function yields a certain value that is a pre-determined threshold. Patterns for which the discriminant function yields a value higher than this threshold are classified as class A, while the rest are classified as class B. Some efforts have already been made to automatically predict lesion [1, 3-14, 20-22, 24-28, 30, 32]. Mass classification algorithms are in continuous development and improvement.

\section{INVESTIGATIONAL ANALYSIS OF MASS CLASSIFIER MODELS}

The classification methods are used to classify suspicious areas of breast DCE-MR images into benign or malignant tissue. The selection of the correct classifier is a factor that very sensitively affects the performance of the correct classification. The selected classifier needs to fit well to the training data (i.e. produce good separation between the classes) while, on the other hand, being as robust as possible to unseen data (generalizability). In this section, Three types of classifiers such as artificial neural networks, support vector machines and bee colony optimization algorithm trained neural network classifier were evaluated for classification of the suspicious lesions in breast MRI and to find an optimal classifier that outperforms all other explored classifiers for the examined dataset of breast DCE -MR images.

\subsection{Artificial Neural Network Classifier}

ANN is a powerful classifier that represents a complex input/ output relationships. It resembles the human brain in acquiring knowledge through learning and storing knowledge within inter-neuron connection strengths. Commonly, the ANN's synaptic weights are adjusted or trained so that a particular input leads to a specific desired or target output. Where the network is adjusted based on comparing neural network output to the desired output until the network output matches the desired output. Once the network is trained it can be used to test new input data using the weights provided from the training session. There were a number of motivations for selecting neural network as a classification mechanism. Neural network have been shown to perform well in medical diagnosis applications [1, 3-9, 20-22, 24- 28].

The proposed artificial neural network classifier model implemented is based on the work discussed in [28], the design includes a three layered neural network, which has an input layer, a hidden layer and an output layer. The number of nodes in the input layer corresponds to the number of input variables, which is seven in this experiment because seven texture features are selected for classification. The linear activation function is used for input layer. The output layer contains one node with values from zero or one indicating the level of malignancy, where zero means absolutely benign and 
one means absolutely malignant. The number of nodes in hidden layer is usually determined by a number of trial-anderror runs. The neural network architecture with different hidden nodes ranging from three to twenty was tested and finally five nodes are selected in the hidden layer. The backpropagation algorithm was used for training, during training; output from the backpropagation neural network (BNN) was compared with the biopsy results (the targets). The mean-square difference was used to estimate the error. Linear transfer function is used for the input layer and hyperbolic tangent sigmoid transfer function for the hidden and output layers neurons.

\subsubsection{Backpropagation Algorithm}

The backpropagation learning algorithm is a supervised learning method that can be used with multilayer networks and nonlinear differentiable transfer functions. Backpropagation is a gradient descent algorithm, in which the network connection weights move along the negative of the gradient of the performance function. The power of backpropagation is that it allows us to calculate an effective error for each hidden unit and thus adjust the hidden weights. As in all supervised learning methods, the backpropagation algorithm consists of presenting an input pattern and changing the network parameters to bring the actual outputs closer to the desired target values. These outputs are compared to the target values; any difference corresponds to an error. This error is some scalar function of the weights, thus the weights are adjusted to reduce the error. This error function is the sum of square differences of the outputs and targets. Let $\mathrm{V}_{\mathrm{i}}$ be the training set elements, $\mathrm{Y}_{\mathrm{i}}$ are the outputs and $\mathrm{O}_{\mathrm{i}}$ are the desired output or targets, then the error function is shown in equation

$$
E=\sum_{i} \sum_{j}\left(y_{j}^{i}-o_{j}^{i}\right)^{2}
$$

The back propagation weight adjustment is done through the equation 2 .

$$
w_{i j}(k+1)=w_{i j}(k)-\eta \frac{\partial E}{\partial w_{i j}}
$$

Where, $\mathrm{k}$ is the iteration number and $\eta$ is the learning rate The weights are adjusted continuously until a better performance is reached.

The MLP artificial neural network training procedure using the Back propagation algorithm thus comprises the following steps:

1. Initialize $\mathrm{W}_{\mathrm{ij}}$ with random small numbers and set $\mathrm{k}=0$.

2. Start with a randomly chosen pattern $\mathrm{v}$ from the training set and evaluate $y$.

3. If $y \neq w$, adjust the weights:

$$
w_{i j}(k+1)=w_{i j}(k)-\eta \delta_{j} z_{i}(k)
$$

Where, $z_{i}(k)$ is node $i$ output and $\delta_{j}$ is the error of the $\mathrm{j}^{\text {th }}$ node.

$$
\delta_{j}=y_{j}\left(1-y_{j}\right)\left(o_{j}-y_{j}\right)
$$

4. Repeat from the second step with another training pattern.

5. $\mathrm{k}=\mathrm{k}+1$.

6. Repeat steps 2-4 until $\|\Delta E(\boldsymbol{W})<\boldsymbol{O}\|$ for all training patterns.

The proposed back propagation algorithm can easily train a neural network for lesion classification data for the prediction task by finding optimal network weights for MLP.

\subsection{Artificial Bee Colony Optimization Algorithm Trained Artificial Neural Network Based Classifier Model}

Artificial neural networks (ANN) are simplified artificial models based on the biological learning process of the human brain. ANN has been very extensive in recent years such as in prognosis and classification. Various researchers have used ANN as a classifier for breast DCE-MR Image mass classification. But the ANN algorithm can get trapped in local minima or it might lead to slow convergence or even network failure. Therefore, neural network alone might not be the best solution. In order to overcome the limitations of standard NN, Artificial bee colony optimisation based techniques have been proposed for MLP.

The neural network classifiers are designed and their structure is individually optimized by an artificial bee colony algorithm based on the work proposed in [29]. The classifier chosen is a multilayer feed forward neural network [19]. The main reason for choosing it is because of its nonparametric statistical property. Unlike the classical statistical classification methods, such as the Bayes classifier, no knowledge of the underlying probability distribution is needed by a neural network. It can learn the free parameters (weights and biases) through training by using examples. This makes it suitable to deal with real problems which are nonlinear, nonstationary and nonGaussian.

Backpropagation (BP) algorithm is accepted learning algorithm used for MLP training [19]. The main task of BP algorithm is to update the network weights for minimising output error using backpropagation processing because the accuracy of any approximation depends on the selection of proper weights for the neural networks (NNs). NNs algorithm can get trapped in local minima or it might lead to slow convergence or even network failure. In order to overcome the drawbacks of standard BP, artificial bee colony optimisation based techniques have been proposed for MLP training. ABC algorithm is an easily understandable technique for training MLP on classification problems [23].

The proposed network architecture consists of a three-layered back-propagation neural network; known as multilayer feed forward artificial neural network (ANN) was utilized to obtain optimal classifier. The three-layered network topology consists of an input layer, a hidden layer and an output layer. The number of nodes in the input layer corresponds to the number of input variables, which is seven in this experiment because seven texture features are selected for classification. The linear activation function is used for input layer. The output layer contains one node with values from zero or one indicating the level of malignancy, where zero means absolutely benign and one means absolutely malignant. The number of nodes in hidden layer is usually determined by a number of trial-and-error runs. The neural network 
architecture with different hidden nodes ranging from three to twenty was tested and finally five nodes are selected in the hidden layer. The standard method for refining such a neural network is using an error backpropagation algorithm. During the training phase, the feedforward calculation is combined with backward error propagation to adjust the weights. In this work the bee's optimization algorithm is used to optimise the weights of the neural network in place of the backpropagation. The Bees algorithm can solve a problem without any information apart from that needed to evaluate fitness. The optimal architecture was chosen as the one for which the validation error was the lowest.

\subsubsection{Neural Network Training using the Bees Algorithm}

The training of a backpropagation neural network involves the minimization of an error component. The error component defines the total difference between the actual output and the desired output of the network over a set of training patterns. Training proceeds by presenting to the network a pattern of known class taken from the training set. The error component associated with that pattern is the sum of the squared differences between the desired and actual outputs of the network corresponding to the presented pattern. The procedure is repeated for all the patterns in the training set and the error components for all the patterns are summed to yield the value of the error function for a backpropagation neural network with a given set of basis function centers, spreads and neuron connection weights.

The optimisation using the bees algorithm will involve the "Bees" searching for the optimal values of the weights assigned to the connections between the neurons within the network each bee represents a neuron in the network with a particular set of basis function centers, spreads and weight vectors. Training an artificial neural network is an optimization task since it is desired to find the optimal set of weights of a neural network in the training process. The aim of the algorithm is to find the bee producing the smallest value of the error function. The algorithm converges to the maximum or minimum without becoming trapped in local optima. The network error function $\mathrm{E}$ is given by equation (5):

$$
E(w(t))=\frac{1}{n} \sum_{j=1}^{n} \sum_{k=1}^{k}\left(d_{k}-O_{k}\right)
$$

Where $\mathrm{E}(\mathrm{w}(\mathrm{t}))$ is the error at the $\mathrm{t}^{\text {th }}$ iteration; $\mathrm{w}(\mathrm{t})$ is the weights in the connections at the $t^{\text {th }}$ iteration; $d_{k}$ is the desired output node; $\mathrm{O}_{\mathrm{k}}$ is the actual value of the $\mathrm{k}^{\text {th }}$ output node; $\mathrm{K}$ is the number of output nodes; and $n$ is the number of patterns. $\mathrm{O}$ is the optimisation target to minimise the objective function by optimizing the network weights $\mathrm{w}(\mathrm{t})$.

The MLP artificial neural network training procedure using the bees algorithm thus comprises the following steps:

\section{1: Initialise the population of solutions $X_{i}$ where $i=1 \ldots . . S N$}

2: Evaluate the fitness of the population. In order to calculate the fitness values of solutions, equation (6)is employed.

$$
\text { fit }_{i}=\left\{\begin{array}{ccc}
\frac{1}{1+f_{i}} & \text { if } & f \geq 0 \\
1+a b s\left(f_{i}\right) & \text { if } \quad f<0
\end{array}\right\}
$$

3: Cycle $=1$

4: Repeat from step 5 to step 8.
5: Apply the training data set to determine the value of the error function associated with each bee. This phase is done by following process.

(i) Produce new solutions $V_{\mathrm{ij}}$ in the neighbourhood of $\mathrm{X}_{\mathrm{ij}}$ for the employed bees using the equation (7).

$$
\mathrm{V}_{\mathrm{ij}}=\mathrm{X}_{\mathrm{ij}}+\Phi_{\mathrm{ij}}\left(\mathrm{X}_{\mathrm{ij}}-\mathrm{X}_{\mathrm{kj}}\right)
$$

(ii) Where $\mathrm{k}$ is a solution in the neighbourhood of i, $\Phi$ is a random number in the range $[-1,1]$ and evaluate them. Apply the Greedy Selection process between processes and calculate the probability values pi for the solutions $\mathrm{X}_{\mathrm{i}}$ as in equation (8).

$$
P_{i}=\frac{f i t_{i}}{\sum_{i=1}^{S N} f i t_{i}}
$$

(iii) Produce the new solutions $\mathrm{V}_{\mathrm{i}}$ for the onlookers from the solutions $\mathrm{X}_{\mathrm{i}}$, selected depending on $\mathrm{P}_{\mathrm{i}}$ and evaluate fitness of them.

(iv) Calculate the error value between the target and obtained value.

6: Based on the error value obtained from step 5, create a new population of bees comprising of the best bees in the selected neighborhoods and randomly placed scout bees. This phase is done by following process.

(i) Apply the Greedy Selection process for the onlookers between $x i$ and $V_{i}$ and determine the abandoned solution (source), if exists, replace it with a new randomly produced solution $\mathrm{X}_{\mathrm{i}}$ for the scout bees

(ii) Memorize the best food source position (solution) achieved so far.

7: Stop if the value of the error function has fallen below a predetermined threshold or after completing the number of iterations.

8: Else, return to step 5.

In the proposed $\mathrm{ABC}$ algorithm for training neural network, each cycle of the search for optimized weights consists of three steps after initialization of the colony, food positions and three control parameters in the number of food source positions, which are the number of employed bees or onlooker bees $(n)$, the value of limit, the maximum cycle number $(R)$ for MLP-ABC algorithm. The initialization of weights was compared with output and the best weight cycle was selected by scout bees' phase. The bees (employed bees, onlooker bees) would continue searching until the last cycle to find the best weights for networks. The food source position of which the nectar was neglected by the bees was replaced with a new food source by the scout bees. Every bee (employed bees, onlooker bees) would produce new solution area for the network and the greedy selection would decide the best food source position.

The food area was limited in range $[-1,1]$. It was applied randomly and was initialized for evaluation, which uses rosenbrock function to evaluate the fitness. Every bee (employed bees, onlooker bees) would produce new evaluated solution area for the network and the greedy selection was decided for the best food source position. If the new food source position has equal or better nectar than the old food 
source position, it was replaced with the new food source position in the memory. Otherwise, the old food source position was retained in the memory. The basic idea of $\mathrm{ABC}$ scheme to train MLP is to use agents of bees to search for the best combination of weights for the network. The greedy selection process was applied between two sets of values $\mathrm{Xi}$ and $\mathrm{Vi}$ while the best scout bees were randomly selected.

The proposed frameworks can easily train a neural network for lesion classification data for the prediction task by finding optimal network weights for MLP.

\subsection{Support Vector Machine (SVM) Classifier}

ANNs have proven good classifiers, but they require a large number of samples for training, which is not always true in practice. Support vector machines (SVMs) are based on statistical learning theory and they specialise for a smaller sample number. The SVMs have better generalisation than ANNs and guarantee the local and global optimal solution similar to that obtained by ANN

The support vector machine based classifier model has been built for breast cancer diagnosis is based on work described in [30]. Support vector machines (SVMs) are an emerging area of research in machine learning and pattern recognition [31]. SVMs are a machine learning method for creating a classification function from a set of labelled training data. The basic concept of SVM is to build a hyperplane as the decision surface in such a way that the boundary of separation among positive and negative examples is maximized. The term SVM come from the fact that the input data points in the training set which are closest to the decision surface are called support vectors. When used in classification; SVM maps the input space to higher dimensional feature space and finds a linear separating hyperplane, which separates class members from non-members [30]. The hypersurface is carefully chosen such that its distance to the nearest training data on either side of the surface is maximized. If the constructed hypersurface is not capable of linearly separating the input data, a kernel transformation function is used to map the input data into a different dimensional space (called a feature space) so that it can be linearly separated using standard SVM hypersurface techniques [30].

The training vectors $x i$ and $x j$ (set of input feature vector and predicts) is mapped into a higher dimensional space by a function $\varnothing$. Then SVM constructs a linear separating hyper plane with the maximal margin in this higher dimensional space. Furthermore, kernel functions can be given as $\mathrm{K}$ (xi, xj) $=\varnothing(\mathrm{xi}) \times \varnothing(\mathrm{xj})$. There are number of kernels that can be used in SVM models. They are linear, polynomial, RBF and sigmoid. For this experimental work, linear kernel is used since it is only a 2 class classification problem. The linear kernel function is given by equation (9)

$$
\mathrm{K}\left(\boldsymbol{x}_{\mathrm{i}}, \boldsymbol{x}_{j}\right)=\boldsymbol{x}_{i} \bullet \boldsymbol{x}_{j}
$$

Where $x_{i}$ and $x_{j}$ are input vectors comprised of one of the previously mentioned feature vectors, $\bullet$ is the dot product operation.

Classification of the lesions has been performed as a two-class problem where the two classes are malignant and benign. There were a number of motivations for selecting SVMs as a classification mechanism. The SVMs have been shown to perform well in medical diagnosis applications [32] and have also been shown to perform well when dealing with relatively small training sets [33]. This was particularly appealing given the inherent difficulty in acquiring large amounts of image data devoted exclusively to training. The support vector machines also perform well and classify reasonably quickly on high dimensional data.

\section{EXPERIMENTAL RESULTS AND DISCUSSION}

This section presents the statistical results of applying different intelligent computer assisted mass classifiers for classifying the region of interest (ROI) that contains mass on real breast DCE- MR images. The breast DCE-MR images are used to classify the mass lesions employing the intelligent classification scheme described above was tested over real images received from Kovai Medical Center and Hospital $(\mathrm{KMCH})$. Coimbatore, Tamil Nadu, India [34]. The dataset contains records of 85 patients. The study included 65 malignant and 20 benign histologically-proven lesions. The data is not publically available and is taken on special request by promising of not to disclose it. The breast DCE-MR images used in this study were acquired with patients prone to $1.5 \mathrm{~T}$ scanner with the use of a dedicated surface breast coil array. The imaging protocol included bilateral fat suppressed T1-weighted images in the sagittal plane of $1 \mathrm{~mm}$ slice thickness and a slab interleaved 3D fat suppressed spoiled gradient echo after the injection of contrast.

Results differed by applying different type of classifiers due to the fact that each classifier has its own method for the formulation of the normal and cancerous clusters upon which it decides whether a test ROI is considered cancerous or normal. This paper have evaluated neural networks, support vector machine and artificial bee colony optimization algorithm trained neural networks as a potential mechanism for the designing the classifiers responsible for delineating between malignant and benign breast lesions from DCE-MRI data.

Classifier performance depends greatly on the characteristics of the data to be classified. Identifying a suitable classifier for a given problem is however still more an art than a science. Classifier performance is a function of several factors including the statistical distribution of the training and testing data, classifier the inherent randomness in the training process and the internal structure of the. Comparison is made between artificial network classifier, SVM classifier and ABC trained neural network classifier based on different strategies for classification to determine and signify the best classifier for our dataset of breast magnetic resonance images. The main reason for using SVM classifiers is because of their good generalization and high precision capabilities. The reason of using multilayer ANN is because it is capable of tolerating the noise, distortion and incompleteness of the data.

The detection performance of the proposed classifiers is measured quantitatively by computing the sensitivity and specificity on the data. Sensitivity (SN) is the proportion of actual positives which are correctly identified and it is mathematically defined in equation (10) and specificity (SP) is the proportion of negatives which are correctly identified and is mathematically defined in equation (11).

$$
\begin{array}{ll}
\text { Sensitivity, } & S N=\frac{T P}{T P+F N} \\
\text { Specificity, } & S P=\frac{T N}{T N+F P}
\end{array}
$$


Accuracy,

$$
A C C=\frac{T P+T N}{T P+F P+F N+T N}
$$

Error in classification,

$$
1 \text { - Accuracy }
$$

Where, TP- true positive, TN- true negative, FP-false positive and $\mathrm{FN}$ - false negative. It is apparent that the main objective of a classifier is to minimize the false positive and negative rates, similarly, to maximize the true negative and positive rates.

The sensitivity, specificity, accuracy and error of the classification technique were evaluated through quantitative measures derived through the comparison of each classified result with its corresponding ground truth. It is defined in equations (10 to 13). Ground truth is based on the diagnosis of the histopathologist.

The three classifiers are designed and their structure is individually optimized such that the performance of the individual classifiers is maximized. In order to evaluate the performance of the proposed three classifier models the breast DCE-MR images are divided into the training set and the test set. The training sets of 50 were used to build the classifier model and the test set of 35 is used to verify the trained classifier model. Note that the cases in the test set are not used to train the classifier model. The obtained optimal results of the proposed three classification algorithms in terms of accuracy, sensitivity and specificity, are reported in Table 1. It has been seen that the SVM classifier has better performance than ANN, but artificial bee colony algorithm optimized neural network based classifier technique shows comparably superior performance than ANN and SVM based classifiers. It should be noted that the same datasets are used by all the classifier algorithms. Results revealed that the optimal feature set which has been extracted by statistical t-test provides high accuracy, sensitivity and high specificity of the available methods than using other features indicates that more diagnostic information about the lesion is available.

An objective method is needed to evaluate the performances of the proposed three mass classification algorithms. The most important performance criterion is accuracy that is the degree to which an algorithm's classification results matches histologically-proven radiologist's classification results. The accuracy of the algorithms is presented in the Table 1 . The sensitivity of the $\mathrm{ABC}$ trained $\mathrm{NN}$ classifier algorithm is high since its accuracy is high and this algorithm is able to provide the same classification of images in all runs which makes it more reliable. The ABC optimized ANN mass classification methodology represented the advantages of a fast learning speed, high efficiency and high accuracy.

Classification performance has been compared in order to determine optimal statistical algorithms for discriminating between disease stages.
Table 1. Detailed analyses of the benign vs. malignant

\begin{tabular}{|c|c|c|c|}
\hline & $\begin{array}{c}\text { ANN } \\
\text { classifier }\end{array}$ & $\begin{array}{c}\text { SVM } \\
\text { Classifier }\end{array}$ & $\begin{array}{c}\text { Bee colony } \\
\text { algorithm } \\
\text { optimized ANN } \\
\text { classifier }\end{array}$ \\
\hline $\begin{array}{l}\text { No of Training } \\
\text { images }\end{array}$ & 50 & 50 & 50 \\
\hline $\begin{array}{l}\text { No of Testing } \\
\text { images (Number of } \\
\text { cases used for } \\
\text { classification) }\end{array}$ & 35 & 35 & 35 \\
\hline True positive & 22 & 23 & 24 \\
\hline True negative & 7 & 7 & 8 \\
\hline False positive & 2 & 2 & 1 \\
\hline False negative & 4 & 3 & 2 \\
\hline Sensitivity (\%) & 84.61 & 88.46 & 92.30 \\
\hline Specificity $(\%)$ & 77.78 & 77.78 & 88.88 \\
\hline Accuracy (\%) & 82.86 & 85.71 & 91.42 \\
\hline $\begin{array}{l}\text { Error in } \\
\text { classification }(\%)\end{array}$ & 17.14 & 14.29 & 8.58 \\
\hline $\begin{array}{l}\text { No of cases } \\
\text { misdiagnosed }\end{array}$ & 6 & 5 & 3 \\
\hline
\end{tabular}
characterization of mass classifier algorithms

Artificial neural networks (ANN) have the advantages mainly of more tolerance to noise inputs and representation of Boolean function apart from others. But too many parameters may result in over fitting. However in support vector machines over fitting is unlikely to occur. The training speed in the neural networks depends on network structure, learning rate, momentum rate and converging criteria. In SVM it depends on the training data size and class separability. The performance of SVMs depends on the selection of kernel type and kernel parameters. The main drawback of SVM is the high algorithmic complexity and extensive memory requirements. The ANN algorithm can get trapped in local minima or it might lead to slow convergence or even network failure. Therefore, neural network alone might not be the best solution. In order to overcome the limitations of standard NN, artificial bee colony optimisation based techniques have been proposed for MLP training and, in the ABC optimized ANN mass classification methodology the training of an ANN is achieved using $\mathrm{ABC}$ optimization algorithm. The synaptic weights of an $\mathrm{ANN}$ are evolved by means of $\mathrm{ABC}$ algorithm. Furthermore, the connections among the neurons that belong to the ANN are synthesized. This allows the generating ease design of the ANN with a high performance. It has been proved that this novel technique is a good optimization algorithm because it does not easily traps in local minima. In general, the results were satisfactory. This mass classifier model allows searching the best values that permit automatically generates a good solution for a classification problem. This classifier outperforms all other existing classifiers. 
Examining the performance evaluation results obtained, it's found that the best results obtained when using artificial bee colony optimization algorithm trained neural network classifier. The experimental results exhibit that the proposed algorithm therefore confirms the usefulness of the algorithm as an optimisation tool and show that the artificial bee colony algorithm is very successful on optimization of training neural network. The proposed mass classifier model is implemented using Matlab 7.5. The computerized texture analysis may therefore provide useful information for reducing the number of negative biopsies. Further investigation will be conducted with a larger data set to determine the generalizability of these results.

\section{CONCLUSION}

A comparison of three different classifier algorithms used in a computer assisted evaluation (CAE) system for breast DCEMRI mass classification has been presented. The algorithms classify starting from seven statistical textural features extracted by t-test from ROI pixel grey levels. The algorithms implemented and tested as classifiers are: artificial neural network, support vector machine and the artificial bee colony algorithm optimized neural network based classifier. The best results are obtained with artificial bee colony algorithm optimized neural network based classifier, which outperform other classification algorithms. Since it achieves accuracy rates of over $91.42 \%$ and experimental results illustrate that $\mathrm{ABC}$ algorithm is flexible and simple to use optimization algorithm. This will lead to a natural development of a CAE system capable of assisting health professionals in the painstaking task of tracing breast DCE-MRI in search of mass abnormalities. The proposed work can be applied to other biomedical image analysis applications.

\section{ACKNOWLEDGEMENT}

The authors would like to thank Dr. R. Rupa, DMRD, DNB, Consultant Radiologist, Kovai Medical Center and Hospital $(\mathrm{KMCH})$; Coimbatore, Tamil Nadu, India, for providing the breast DCE-MR images used for testing the algorithms proposed and her valuable clinical advice.

\section{REFERENCES}

[1] Nirooei M, Abdolmaleki P, Tavakoli A and Gity M. "Feature selection and classification of breast cancer on dynamic magnetic resonance imaging using genetic algorithm and artificial neural networks". J.Electrical Systems. Vol: 5(1) , 2009.

[2] L. Liberman EA. Morris MJ. Lee, JB. Kaplan, LR. LaTrenta JH. Menell, AF. Abramson, SM. Dashnaw, DJ. Ballon, DD. Dershaw. "Breast lesions detected on MR Imaging: Features and positive predictive value". J. American Roentgen Ray Society. Vol: 179, pp. 171-178, 2002.

[3] Lucht R, Delorme S, Brix G. "Neural network-based segmentation of dynamic MR mammographic images". Magnetic Resonance Imaging. Vol: 20, 2002, pp: 147154

[4] Degenhard A, et al. "Comparison between radiological and artificial neural network diagnosis in clinical screening. Physiological Measurement.Vol:23, 2002, pp: 727-739.

[5] Lucht RE, Knopp MV, Brix G. "Classification of signaltime curves from dynamic MR mammography by neural networks". Magnetic Resonance Imaging. Vol: 19, 2001, pp: $51-57$.
[6] Fischer H, Hennig J. "Neural network-based analysis of MR time series". Magnetic Resonance in Medicine. Vol: 41, 1999, pp: 124-131.

[7] Tzacheva AA, Najarian K, Brockway JP. "Breast cancer detection in gadolinium-enhanced MR images by static region descriptors and neural networks". Journal of Magnetic Resonance Imaging.Vol:17, 2003, pp: 337 342 .

[8] Szabo BK, Aspelin P, Wiberg MK."Neural network approach to the segmentation and classification of dynamic magnetic resonance images of the breast: comparison with empiric and quantitative kinetic parameters". Academic Radiology. Vol: 11, 2004, pp: $1344-1354$

[9] Vomweg TW, et al. "Improved artificial neural networks in prediction of malignancy of lesions in contrastenhanced MR-mammography". Medical Physics. Vol: 30(9), 2003, pp: 2350-2359.

[10] Jacobs MA, et al. "Benign and malignant breast lesions: diagnosis with multiparametric MR imaging". Radiology. Vol: 229, 2003, pp: 225-232.

[11] Gilhuijs KGA, Giger ML. "Computerized analysis of breast lesions in three dimensions using dynamic magnetic-resonance imaging”. Medical Physics. Vol: 25, 1998, pp: 1647-1654.

[12] Chen W, Giger ML, Lan L, Bick U. "Computerized interpretation of breast MRI: Investigation of enhancement-variance dynamics". Medical Physics. Vol: 31(5), 2004, pp: 1076-1082

[13] Nattkemper TW, et al. "Evaluation of radiological features for breast tumour classification in clinical screening with machine learning methods". Artificial Intelligence in Medicine. 2005;34:129-139.

[14] Levman J, Plewes DB, Martel AL. "Validation of SVM based classification of DCE-MRI breast lesions". Proceedings MICCAI 2006 Workshop on Medical Image Processing: The Challenges in Clinical Oncology; Copenhagen, DK. October 2006.

[15] Wael A. Mohamed, Yasser M. Kadah. "Computer Aided Diagnosis of Digital Mammograms". International conference on computer engieering and systems, ICCES 07, 2007 pp: 299-303.

[16] Tuceryan, $M$ and Jain, A.K., "Texture analysis, in the handbook of pattern recognition and computer vision". ( $2^{\text {nd }}$ Edition), World Scientific Publishing Co. 1998, p. 207-248.

[17] Liapis S, Sifakis E and Tziritas, G. "Colour and texture segmentation using wavelet frame analysis, deterministic relaxation, and fast marching algorithms". J. Vis. Commun. Image. Vol: 15, 2004, pp. 1-26.

[18] Robert M. Haralick, K. Shanmugam and Itshak Dinstein, "Textural features for image Classification". IEEE Transaction on systems, man and cybernetics, vol.smc-3, No. 6, November 1973.

[19] Ionna christoyianni, athanasios koutras, evangelos dermatas and george kokkinakis, "Breast tissue classification in mammograms using ICA mixture models". Artificial neural networks, ICANN 2001. 
[20] Bahreini L, Fatemizadeh E, and Gity M. "Gradient vector flow snake segmentation of breast lesions in dynamic contrast-enhanced MR images". The 17th Iranian Conference on Biomedical Engineering (ICBME), 2010, Nov 3-4; Isfahan, Iran.

[21] Arbash-Meinel L, Stolpen AH, Berbaum KS, et al. "Breast MRI lesion classification: Improved performance of human readers with a backpropagation neural network computer-aided diagnosis (CAD) system". J Magn Reson Imaging. Vol: 25(1), 2007, pp: 89-95.

[22] L. Arbach, A. Stolpen, and J.M. Reinhardt, "Classification of breast MRI lesions using a backpropagation neural network (BNN)." $2^{\text {nd }}$ IEEE International Symposium on Biomedical Imaging: Macro to Nano, Piscataway, NJ (2004).

[23] Karaboga, D. "An idea based on honey bee swarm for numerical optimization". Technical Report-TR06; Engineering Faculty, Computer Engineering Department, Erciyes University: Kayseri, Turkey, (2005)

[24] Christine E. McLaren, Wen-Pin Chen, Ke Nie, and MinYing Su. "Prediction of malignant breast lesions from MRI features: A comparison of artificial neural network and logistic regression techniques". Acadamic Radiology. Vol: 16 (7), 2009, pp: 842-851.

[25] Keyvanfard.F, Shoorehdeli. M. A., Teshnehlab M. "Feature selection and classification of breast cancer on dynamic magnetic resonance imaging using ANN and SVM". American Journal of Biomedical Engineering. Vol: 1(1), 2011, pp: 20-25.

[26] P.Abdolmaleki, H.Abrishami-Moghddam, M. Gity, "Improving the performance of neural network in differentiation of breast tumors using wavelet transformation on dynamic MRI". Iran. J. Radiat. Res. Vol: 3 (3), 2005, pp: 135-142.
[27] Erez. Eyal, Daria Badikhi, Edna Furman-Haran, et.al. "Principal component analysis of breast DCE-MRI adjusted with a model based method". J Magn Reson Imaging. Vol: 30 (5), 2009, pp: 989-998.

[28] Janaki sathya D and Geetha K. "Breast MRI lesion classification using backpropagation neural network". Karpagam University $4^{\text {th }}$ Annual Research Congress (Kuarc) 2012, 29-30 November 2012.

[29] Janaki sathya D and Geetha K. "Mass classification in breast DCE-MR images using an artificial neural network trained via a bee colony optimization algorithm". ScienceAsia journal. Vol: 39(3), 2013, pp: 294-305.

[30] Jacob Levman, Tony Leung, Petrina Causer, Don Plewes, and Anne L. Martel, "Classification of dynamic contrast-enhanced magnetic resonance breast lesions by support vector machines". IEEE Trans Med Imaging. Vol: 27(5), 2008, pp: 688-696.

[31] Vapnik VN. The nature of statistical learning theory. New York, NY: Springer; 2000.

[32] Chang Ruey-Feng, Wu Wen-Jie, Moon Woo Kyung, Chou Yi-Hong, Chen Dar-Ren. "Support vector machine for diagnosis of breast tumors on US images". Academic Radiology.Vol:10, 2003, pp:189-197.

[33] El-Naqa I, Yang Y, Wernick MN, Galatsanos NP, Nishikawa RM. "A support vector machine approach for detection of microcalcifications. IEEE Transactions on Medical Imaging". Vol: 21, 2002, pp: 1552-1563.

[34] Medical dataset - Real time, Kovai Medical Center and Hospitals, Coimbatore. 